\title{
Epidemiological investigation and genetic characterization of porcine astrovirus genotypes 2 and 5 in Yunnan province, China
}

\author{
Keqing Ren ${ }^{1} \cdot$ Rong Wang $^{1} \cdot$ Xiao Liu $^{1} \cdot$ Yinghua Liu ${ }^{1}$. Jintao Zhang ${ }^{1} \cdot$ Junyu Bi ${ }^{1} \cdot$ Lianfeng Zhao $^{2} \cdot$ Zhigang Guo $^{2}$. \\ Jianping Liu ${ }^{2}$ (D) Gefen Yin ${ }^{1}$
}

Received: 29 June 2021 / Accepted: 10 October 2021 / Published online: 28 November 2021

(c) The Author(s), under exclusive licence to Springer-Verlag GmbH Austria, part of Springer Nature 2021

\begin{abstract}
Astroviruses (AstVs) are among the most important viruses causing diarrhea in human infants and many animals, posing a threat to public health safety and a burden on the economy. Five porcine AstV (PAstV) genotypes have been identified in various countries, including China. However, the epidemiology of PAstV in Yunnan province, China, remains unknown. In this study, 489 fecal samples from pigs in all 16 prefectures/cities of Yunnan were collected between April and August of 2020 for epidemiological investigation. The total infection rate of PAstV-2 or PAstV-5 was 39.9\%, with suckling piglets having the highest infection rate (62.3\%). The ORF2 genes of seven PAstV-2 and 10 PAstV-5 isolates were sequenced and phylogenetically analyzed. In addition to coinfections with PAstV-2 and PAstV-5, coinfections of PAstV with other diarrheainducing viruses (e.g., porcine bocavirus) were also discovered. A comparison of ORF2-encoded capsid protein sequences revealed that there were multiple insertions and deletions in the seven Yunnan PAstV-2 sequences, while point mutations, but no deletions or insertions, were found in the 10 Yunnan PAstV-5 sequences, which were very similar to the reference sequences. This is the first epidemiological investigation and genetic characterization of PAstV-2 and PAstV-5 in Yunnan province, China, demonstrating the current PAstV infection situation in Yunnan.
\end{abstract}

\section{Introduction}

Astroviruses (AstVs) are among the most important viruses causing diarrhea in human infants and numerous animals worldwide [1, 2], with outbreaks occurring predominantly in spring and winter [3]. AstVs are small, non-enveloped, positive-sense single-stranded RNA viruses that belong to the family Astroviridae, with genomes of 6-8 kb in length and viral particles of $28-35 \mathrm{~nm}$ in diameter, characterized by 5- to 6-pointed star-like surfaces [4]. The genome of AstVs

Handling Editor: Akbar Dastjerdi.

Keqing Ren and Rong Wang contributed equally to this work.

Jianping Liu

liujianping88@hotmail.com

$\triangle$ Gefen Yin

yingefen0616@163.com

1 College of Animal Veterinary Medicine, Yunnan Agricultural University, Kunming 650201, Yunnan, China

2 College of Life Sciences, Nanjing Normal University, Wenyuan Rd. No. 1, Nanjing 210023, Jiangsu, China comprises a $5^{\prime}$ noncoding region, three open read frames (ORF1a, ORF1b, and ORF2), a 3' noncoding region, and a polyA region [4]. ORF2 encodes the capsid protein, a viral immune-related protein with high immunogenicity that varies in structure between different species and genotypes [5]. The region including amino acids 1-415 (N-terminus) of the capsid protein is highly conserved, and the region starting at amino acid 416 (C-terminus) is extremely variable and is the neutralizing antigenic determinant of the virus, with multiple neutralization epitopes [6]. Based on their ORF2 gene sequences and host specificity, AstVs are classified into 3 species in the genus Avastrovirus, whose members infect birds, and 19 species of the genus Mamastrovirus, whose members infect mammals. Mamastroviruses that infects pigs (porcine astroviruses, PAstVs) are classified into five genotypes (PAstV1-5) based on phylogenetic analysis of the fulllength ORF2 capsid protein sequence, regardless of clinical manifestations [7-9].

Since its first detection by electron microscopy in the feces of piglets with diarrhea in 1980 [10] and its first isolation from a case of porcine acute gastroenteritis in 1990 [11], PAstV has been prevalent in many countries, including Kenya and Uganda [12], the USA [9, 13-20], Canada 
[7], Colombia [21, 22], Croatia [23], Italy [24], Belgium [25], Austria [26], Hungary [27-29], India [30], Thailand [31], South Korea [32, 33], and Japan [34]. There have been many reports of PAstV infections in China, with all five PAstV genotypes present in Guangxi [35-37], PAstV-1, -2, -4 , and -5 in Hunan $[38,39]$, PAstV-2 and -5 in Sichuan [40], PAstV-2 in Shanghai [41], PAstV-5 in Jilin [42], and PAstV-4 in Anhui [43] and Tianjin [44]. However, there has been no report on PAstV epidemiology in Yunnan, one of China's top porcine-protein-producing provinces, with a turnover of approximate 31.45 million pigs, including 3.2 million sows.

In the present study, 489 fecal samples were collected from various pig populations, with or without diarrhea, from all 16 prefectures/cities in Yunnan province between April and August of 2020 to investigate the prevalence of PAstV infections in Yunnan. The ORF2 gene sequences of viruses found in these samples were genetically characterized to provide insights into the epidemiology and genetic relationships of PAstVs circulating in Yunnan for better monitoring, early warning, prevention, and control of PAstV.

\section{Materials and methods}

\section{Sample collection}

Four hundred eighty-nine separate pig fecal samples were collected from all 16 prefectures/cities of Yunnan province, China, between April and August of 2020 (Table 1). The sampled pigs were differentiated as suckling piglets, weaned piglets, finisher pigs, and sows, and roughly equal numbers of samples were collected from each $(130,139,117$, and 103 , respectively) (Table 1). The number of samples from each prefecture or city was proportional to the size of the farms and depended on the convenience of sampling. Among the samples, approximately one-third (151 samples) were from pigs with diarrhea, while the remaining two-thirds (338 samples) were from pigs without clinical signs of diarrhea. Fecal samples were collected with sterile swabs and shipped in sterile $15-\mathrm{mL}$ Falcon tubes on ice to the lab for storage at $-80^{\circ} \mathrm{C}$ until viral nucleic acid isolation.

\section{Sample processing and viral nucleic acid extraction}

Approximately $5 \mathrm{~mL}$ of $0.85 \%$ sterile saline was added to each $15-\mathrm{mL}$ Falcon tube, followed by thorough mixing and incubation at $4{ }^{\circ} \mathrm{C}$ overnight. Two $\mathrm{mL}$ of the suspension was then subjected to centrifugation at $8000 \mathrm{rpm}$ for $10 \mathrm{~min}$. Four hundred microliters of supernatant was used for viral RNA and DNA extraction, using TRIpure Total RNA Extraction Reagent (cat. no.: RP001, BioTeke, Beijing, China) and a FinePure DNA Extraction Kit (cat. no.:
Table 1 Information about samples used in this study

\begin{tabular}{|c|c|c|c|c|}
\hline Prefecture/city & $\begin{array}{l}\text { Suckling } \\
\text { piglets }\end{array}$ & $\begin{array}{l}\text { Weaned } \\
\text { piglets }\end{array}$ & Finisher pigs & Sows \\
\hline Baoshan & 13 & 11 & 9 & 3 \\
\hline Chuxiong & 6 & 8 & 10 & 3 \\
\hline Dali & 9 & 8 & 5 & 7 \\
\hline Dehong & 12 & 18 & 15 & 11 \\
\hline Diqing & 4 & 8 & 5 & 9 \\
\hline Honghe & 5 & 7 & 8 & 6 \\
\hline Kunming & 12 & 9 & 7 & 7 \\
\hline Lijiang & 5 & 8 & 6 & 5 \\
\hline Lincang & 6 & 4 & 5 & 4 \\
\hline Nujiang & 10 & 8 & 7 & 6 \\
\hline Pu'er & 5 & 3 & 7 & 9 \\
\hline Qujing & 8 & 10 & 5 & 4 \\
\hline Wenshan & 9 & 7 & 3 & 2 \\
\hline $\begin{array}{l}\text { Xishuang- } \\
\text { banna }\end{array}$ & 8 & 12 & 9 & 10 \\
\hline Yuxi & 11 & 10 & 6 & 8 \\
\hline Zhaotong & 7 & 8 & 10 & 9 \\
\hline Sum & 130 & 139 & 117 & 103 \\
\hline Sum & 489 & & & \\
\hline
\end{tabular}

DP1902, BioTeke, Beijing, China), respectively, according to the manufacturer's instructions. The remaining sample was kept at $-80^{\circ} \mathrm{C}$ until further use.

\section{Detection of selected porcine viruses and cloning of the PAstV ORF2 fragment}

The detailed protocol is available upon request. In brief, cDNA synthesis was performed using the 489 RNA samples and EasyScript RT/RI Enzyme Mix (cat. no. AE31102, Transgen, Beijing, China) and the downstream primer PAstV-2R or PAstV-5R for detection of PAstV-2 or PAstV5 , respectively. The oligonucleotides were purchased from Tsingke Biotech, Kunming, China, and their sequences are shown in Table 2). This was followed by PCR using $2 \times$ Phanta Max Master Mix (cat. no. P505-01, Vazyme, Nanjing, China) and the primer pairs listed in Table 2 (PAstV2F/PAstV-2R and PAstV-5F/AstV-5R, respectively). PAstVpositive samples were identified by gel electrophoresis to visualize the specific amplification products.

The PAstV-positive samples were then tested for another nine diarrhea-related porcine viruses, namely, porcine epidemic diarrhea virus (PEDV), transmissible gastroenteritis coronavirus (TGEV), porcine rotavirus (PoRV), porcine bocavirus $(\mathrm{PBoV})$, porcine sapovirus $(\mathrm{PoSaV})$, porcine deltacoronavirus (PDCoV), classical swine fever virus (CSFV), pseudorabies virus (PRV), and porcine circovirus 2 (PCV2). Based on the corresponding reference sequences, 
Table 2 Primers used in this study

\begin{tabular}{|c|c|c|c|c|c|}
\hline Virus & Primer name & Primer sequence $\left(5^{\prime} \rightarrow 3^{\prime}\right)$ & Amplified region & $\begin{array}{l}\text { Product } \\
\text { length (bp) }\end{array}$ & Reference sequence no. \\
\hline \multirow[t]{8}{*}{ PAstV } & PAstV-2F & CCTGATGCACAACAGTGAAG & ORF1b-ORF2 & 203 & LC201589 \\
\hline & PAstV-2R & ATTGCGCACCACGATGTTGGT & & & \\
\hline & PAstV-5F & CAACAGCTCTTGCGCATTGTG & ORF1b & 333 & KP747574 \\
\hline & PAstV-5F & GATGTCATCAGGGTCAGGAC & & & \\
\hline & PAstV2ORF2-F & ACTGATGAGCAGCTGGATCGT & ORF2 & 2505 & MK460230 \\
\hline & PAstV2ORF2-R & CATGACGCGAGTGCCATCCTA & & & \\
\hline & PAstV5ORF2-F & CGATGGCCAATCGGCGTAACAG & ORF2 & 2248 & JF713711 \\
\hline & PAstV5ORF2-R & ATGAGCTGTACCCTCGGTCCTA & & & \\
\hline \multirow[t]{2}{*}{ PEDV } & PEDV-F & TTTATTCTGTCACGCCATGT & spike & 420 & MN412572 \\
\hline & PEDV-R & CTATGGTCTTACATGCTGCAG & & & \\
\hline \multirow[t]{2}{*}{ TGEV } & TGEV-F & TTGACTTGCAATTGGGGTAG & spike & 425 & MN510432 \\
\hline & TGEV-R & GAGCTATTAGTTAGAAGGAAC & & & \\
\hline \multirow[t]{2}{*}{ PoRV } & PoRV-F & CCTGGTCCATTCGCACAAAC & VP4 & 475 & MK283695 \\
\hline & PoRV-R & CATAGTTAGTAGTCGAATAG & & & \\
\hline \multirow[t]{2}{*}{ PBoV } & PBoV-F & AGCGTCTAGGTAAGAAGCC & NP1 & 326 & MG846651 \\
\hline & PBoV-R & TCATTCGGTCTCCTCCATGTC & & & \\
\hline \multirow[t]{2}{*}{$\mathrm{PoSaV}$} & PoSaV-F & CCCTCATTGGACCAAGTGGGA & VP1 & 615 & MK965898 \\
\hline & PoSaV-R & ACACTGTGTAAGGTTCGGTAC & & & \\
\hline \multirow[t]{2}{*}{ PDCoV } & Delta-F & ATCCTCCAAGGAGGCTATGC & $\mathrm{N}$ & 554 & МH025764 \\
\hline & Delta-R & GAAGTGGTTATGGTGTGAAG & & & \\
\hline \multirow[t]{2}{*}{ CSFV } & CSFV-F & GTGGAGGAACCGGTATATGATG & Polyprotein & 360 & MN399384 \\
\hline & CSFV-R & CCGTCACTACCTGTCACCCTAC & & & \\
\hline \multirow[t]{2}{*}{ PCV2 } & PCV2-F & CGAACGCAGTGCCGAGGCCT & ORF2 & 607 & MW262924 \\
\hline & PCV2-R & ATGACGTATCCAAGGAGGCGTT & & & \\
\hline \multirow[t]{2}{*}{ PRV } & PRV-F & CCGCGGGCCGTGTTCTTTGT & $\mathrm{gE}$ & 501 & JQ809328 \\
\hline & PRV-R & CGTGGCCGTTGTGGGTCAT & & & \\
\hline
\end{tabular}

the primers used in this study were designed using the online primer-design software Primer3web (version 4.1.0) [45] and are listed in Table 2. The viral DNA (for PBoV and PCV2) or RNA (for the other seven viruses) was detected by PCR using 2× TransTaq HiFi PCR Super Mix II (cat. no. AS13121, Transgen, Beijing, China) or RT-PCR (see above) and gel electrophoresis.

To determine the full-length ORF2 gene sequences of the PAstV-2 or PAstV-5 isolates from the positive samples, the PCR products obtained using the primer pairs listed in Table 2 (PAstV2ORF2-F/PAstV2ORF2-R and PAstV5ORF2-F/PAstV5ORF2-R, respectively) were gelpurified, cloned into the vector pMD18-T (cat. no. 6011, Takara, Dalian, China), purified, and used to transform $E$. coli $\mathrm{DH} 5 \alpha$ for bulk culture and subsequent Sanger sequencing at Sangon Biotech (Shanghai, China).

\section{Sequence analysis}

ORF2 codes for a capsid protein whose sequence is extremely variable due to immune pressure from the host and is therefore commonly used for genetic classification of AstV isolates [46]. Selected PAstV ORF2 gene sequences (Supplementary Table S1) were retrieved from the GenBank database for sequence alignments and phylogenetic analysis with the ORF2 gene sequences determined in this study. DNAStar 6.0 software was used with default parameters to assemble the sequences of ORF2 fragments from Yunnan and to compare the ORF2 gene and capsid protein amino acid sequences of the selected reference sequences (Supplementary Table S1) and the sequences obtained in this study (Supplementary Table S2). A phylogenetic tree based on the aligned nucleotide sequences was constructed, and a multiple amino acid sequence alignment for indel (inserts and deletions) analysis was made using the ClustalW alignment program included in the MEGA 7.0 software package [47]. 


\section{Results}

\section{Molecular detection of PAstV-2 and PAstV-5 in Yunnan province, China}

PAstV is an important pathogen causing diarrhea in piglets and has been widely distributed in China for more than 10 years. In particular, PAstV-2 and PAstV-5 are predominant, as exemplified by a report of the complete genome sequence of an isolate from Shanghai in 2012 [48] and epidemiological investigations in Guangxi between 2013 and 2015 [35], in Sichuan in 2014 [40], in 17 provinces or municipalities in China between 2015 and 2018 [49], and in Hunan in 2017 [39]. However, to our best knowledge, there has not been an epidemiological study in Yunnan, one of the most important swine-producing provinces in China, which adjoins several PAstV-prevalent areas (e.g., Sichuan and Guangxi) and shares long geographic borders with Vietnam, Laos, and Myanmar.

Primer pairs specific for PAstV-2 and PAstV-5 (PAstV2F/2R and PAstV-5F/5R, Table 2) were designed for RTPCR to amplify the PAstV-2 ORF1b-ORF2 and PAstV-5 ORF1b fragments from 489 fecal samples collected in Yunnan province between April and August of 2020. Gel electrophoresis of the amplification products (data not shown) revealed that, out of the 489 samples, there were 107 PAstV-2-positive samples (amplicon length, $203 \mathrm{nt}$ ) and 92 PAstV-5-positive samples (amplicon length, 333 nt). As summarized in Table 3 , the PAstV-2 positivity rate in clinically diarrheal pigs $(46 / 151,59.0 \%)$ was moderately higher than that in non-diarrheal pigs $(61 / 338,52.1 \%)$, while no obvious difference in the PAstV-5 positivity rate was observed between clinically diarrheal pigs $(36 / 151$, $46.2 \%)$ and non-diarrheal pigs $(56 / 338,47.9 \%)$. Considering that both PAstV-2 and PAstV-5 were detected in the same four diarrheic fecal samples (YN-158, YN-224, YN-328, and YN-587) collected from two suckling piglets and two weaned piglets, the overall PAstV infection rate for diarrheic and non-diarrheic fecal samples was $51.7 \%$ and $34.6 \%$, respectively (Table 3 ).

As shown in Fig. 1A, the PAstV-positive samples $(195 / 489,39.9 \%)$ were distributed across all 16 prefectures/ cities in Yunnan, with the positive rates ranging between $14.8 \%$ (4/27, Chuxiong) and 74.1\% (20/27, Qujing). One hundred ninety-five PAstV-positive samples were detected in all four pig populations included in this investigation (Fig. 1B), with the positivity rate ranging from $12.3 \%$ (finisher pigs) to $41.5 \%$ (suckling piglets). The data in Table 3 show that the positivity rates for PAstV-2 were higher than those for PAstV-5 in three of the four pig groups (54.3\% vs. $48.1 \%$ in suckling piglets, $56.3 \%$ vs. $46.9 \%$ in weaned piglets, and $57.7 \%$ vs. $42.3 \%$ in finisher pigs), while no difference was observed in sows (50\% vs. 50\%). Overall, piglets appear to be much more vulnerable to PAstV infection (62.3\% for suckling piglets and $46.0 \%$ for weaned piglets) than finisher pigs (22.2\%) and sows (23.3\%) (Table 3).

\section{Coinfection of PAstV with multiple porcine viruses in Yunnan}

Coinfections with multiple porcine pathogens are common in pigs, often leading to more clinical severity $[50,51]$. PAstV has been identified as an important agent of diarrhea [36] and frequently coinfects with other porcine pathogens $[31,40]$. Consequently, there was a trend that individuals with poor body condition (e.g., due to infections with other viruses) had a higher probability of shedding astroviruses in their feces [3]. Therefore, we decided to monitor coinfections of PAstV with other porcine viruses in Yunnan.

In our current study, PAstV coinfections with nine porcine viruses were investigated using established protocols for detection of PEDV, TGEV, PoRV, PBoV, PoSaV, PDCoV, CSFV, PRV, and PCV2. Of the 195 PAstV-positive fecal samples, 71 (36.4\%) contained only PAstV, while the other $124(63.6 \%)$ contained at least one of the nine selected porcine viruses, with infection rates ranging from $0.5 \%(1 / 195$ for PDCoV) to $36.4 \%$ (71/195 for PBoV) (Fig. 1C). Of these

Table 3 PAstV-2 and -5 infections in the four pig populations and in samples from pigs with or without diarrhea

\begin{tabular}{|c|c|c|c|c|c|c|c|}
\hline \multirow[t]{2}{*}{ Pig population } & \multirow{2}{*}{$\begin{array}{l}\text { Total number of } \\
\text { samples }\end{array}$} & \multicolumn{2}{|l|}{ PAstV-2 } & \multicolumn{2}{|l|}{ PAstV-5 } & \multicolumn{2}{|l|}{ Total } \\
\hline & & $\begin{array}{l}\text { Positive } \\
\text { samples }\end{array}$ & Positive rate (\%) & $\begin{array}{l}\text { Positive } \\
\text { samples }\end{array}$ & Positive rate (\%) & $\begin{array}{l}\text { Positive } \\
\text { samples }\end{array}$ & Positive rate $(\%)$ \\
\hline Suckling piglets & 130 & 44 & 54.3 & 39 & 48.1 & 81 & 62.3 \\
\hline Weaned piglets & 139 & 36 & 56.3 & 30 & 46.9 & 64 & 46.0 \\
\hline Sows & 103 & 12 & 50.0 & 12 & 50.0 & 24 & 23.3 \\
\hline Finisher pigs & 117 & 15 & 57.7 & 11 & 42.3 & 26 & 22.2 \\
\hline Diarrhea & 151 & 46 & 59.0 & 36 & 46.2 & 78 & 51.7 \\
\hline Non-diarrhea & 338 & 61 & 52.1 & 56 & 47.9 & 117 & 34.6 \\
\hline Total & 489 & 107 & 54.9 & 92 & 47.2 & 195 & 39.9 \\
\hline
\end{tabular}




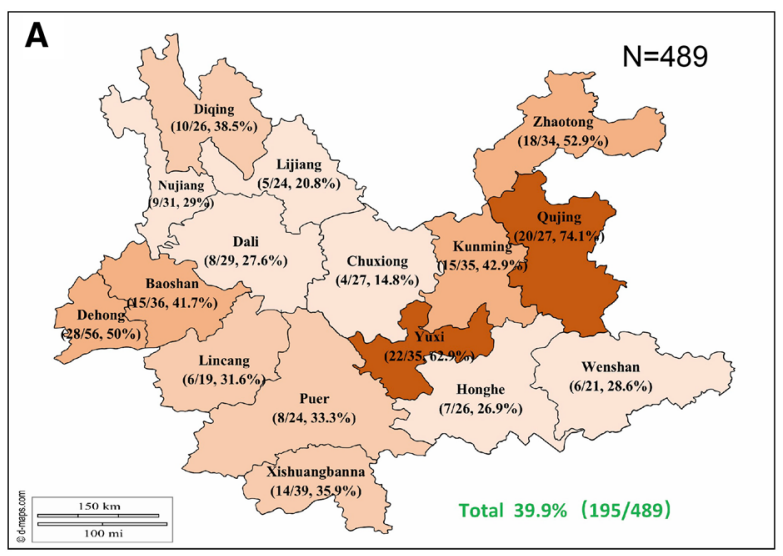

C

$N=195$

$36.4 \%$

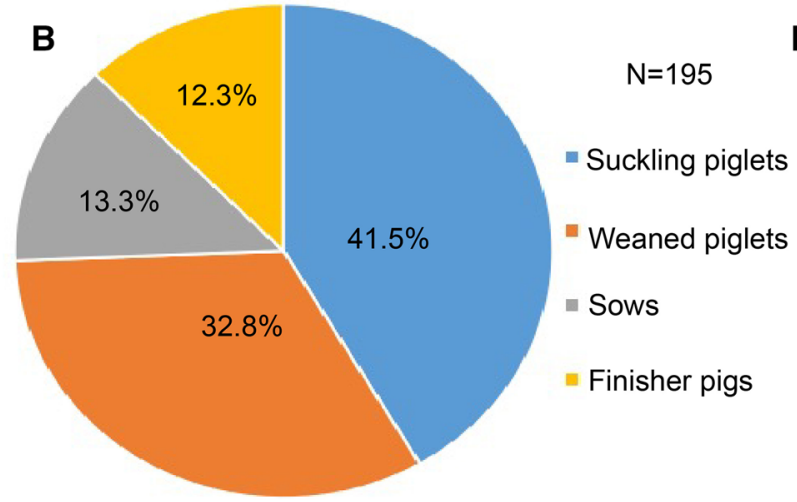

PBOV POSaV PCV2 PORV PEDV CSFV PRV TGEV PDCOV

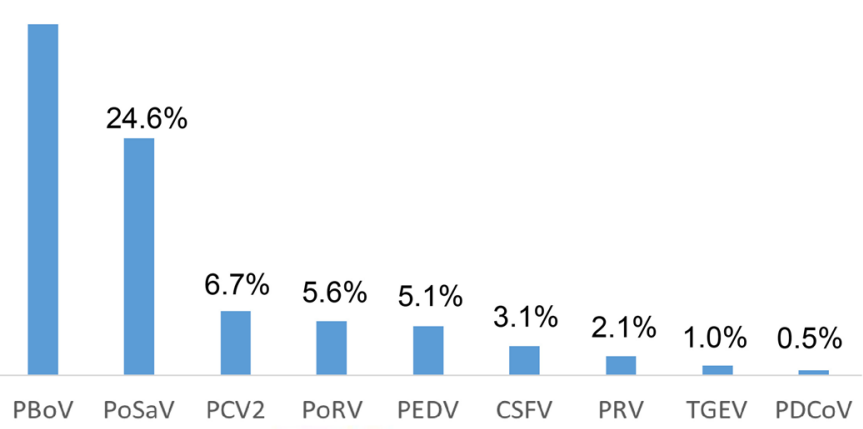

D

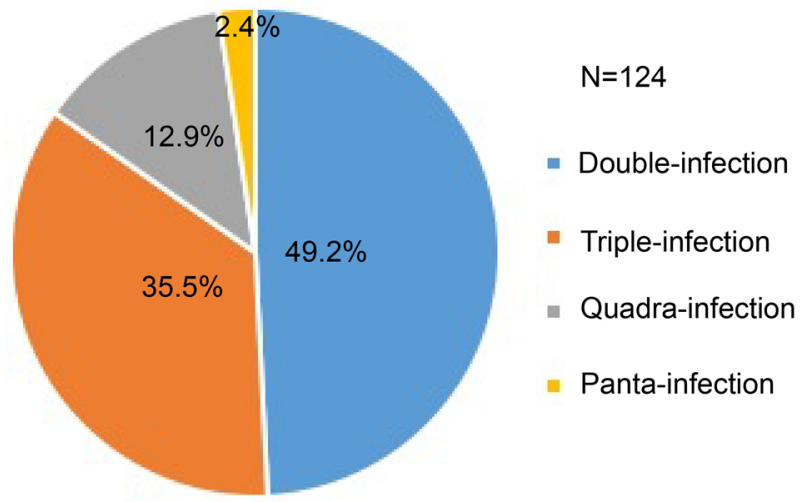

Fig. 1 Epidemiological investigation of porcine astrovirus (PAstV) infections in Yunnan province, China. Testing of 489 fecal samples by RT-PCR using genotype-specific primers against PAstV-2 or PAstV-5 revealed that PAstV infection is endemic in Yunnan. (A) Locations of PAstV infections in Yunnan. Prefectures/cities with a PAstV infection rate above $60 \%$ are highlighted in brown, $40-60 \%$ in light orange, $30-40 \%$ in beige, and below $30 \%$ in light beige. (B) Pie

co-infected 124 samples, 49.2\% (61/124) contained PAstV and one other virus, while 35.5\% (44/124) contained PAstV and two other viruses, $12.9 \%$ (16/124) contained PAstV and three other viruses, and 2.4\% (3/124) contained PAstV and four other viruses (Fig. 1D). Coinfections with PAstV and PoRV, PEDV, TGEV, CSFV, or PCV2 have been reported previously [15, 30, 31, 40, 42, 49]. In the present study, $\mathrm{PBoV}$ was the most frequently found coinfecting virus in PAstV-positive samples (36.4\%, 71/195).

\section{Phylogenetic analysis of PAstV-2 and PAstV-5 ORF2 gene sequences from Yunnan}

Information on the genetic characteristics of PAstV in China is still rather limited. Therefore, it is necessary to investigate the genetic diversity and relationships of PAstV strains currently circulating in Yunnan. In the current study, in addition to the four samples from animals with diarrhea (YN224, YN328, YN-510, and YN587) that were simultaneously infected with both PAstV-2 and PAstV-5, six PAstV-2- and

chart distribution of the 195 PAstV-positive fecal samples among the four pig groups (suckling piglets, weaned piglets, sows, and finisher pigs). (C) Coinfection with nine selected swine diarrhea-inducing viruses in the 195-PAstV positive fecal samples. (D) Pie chart distribution of the multiple infections with PAstV in the 124 coinfected fecal samples.

six PAstV-5-positive samples were randomly selected for determination of the complete ORF2 gene sequence using the primer pairs PAstV2ORF-F/PAstV2ORF-R and PAstV5ORF-F/PAstV5ORF-R (Table 2), respectively. A total of seven PAstV-2 ORF2 genes and 10 PAstV-5 ORF2 genes, 2251-2402 nt long, were successfully amplified, cloned, and sequenced from the 16 selected PAstV-positive samples. Interestingly, ORF2 gene sequences were detected in all four diarrheic fecal samples that were positive for both PAstV-2 and PAstV-5. The 17 PAstV ORF2 gene sequences obtained in this study were submitted to the GenBank database under the accession numbers MZ325424-MZ325440 (Supplementary Table S2) and are shown in the file 'Supplementary Sequences'.

A phylogenetic tree (Fig. 2) was constructed based on the 17 PAstV ORF2 gene sequences from this study (Supplementary Table S2) and 40 selected reference PAstV ORF2 gene sequences (Supplementary Table S1): three for PAstV-1, thirteen for PAstV-2, seven for PAstV-3, eight for PAstV-4, and nine for PAstV-5. The $57 \mathrm{PAstV}$ strains were 
Fig. 2 Phylogenetic analysis of PAstV-2 and PAstV-5 strains from Yunnan based on ORF2 gene sequences. Seventeen PAstV ORF2 gene sequences were determined to be 2208$2343 \mathrm{nt}$ in length and compared with 40 selected PAstV reference sequences (Supplementary Table S1) using the software MEGA 7.0 [47]. The phylogenetic tree revealed that seven PAstV ORF2 sequences from this study (YN-51, YN-107, YN-158, YN224-2, YN328-

2, YN-510-2, and YN587-2) belong to the PAstV-2 genotype, while the other $10 \mathrm{PAstV}$ sequences (YN-54, YN-92, YN-120, YN-224-5, YN-328-5, YN-336, YN387, YN-510-5, YN-550, and YN-587-5) belong to the PAstV-5 genotype. Both PAstV-2 and PAstV-5 genotypes were simultaneously identified in four diarrheic stool samples (indicated by a green square), suggesting coinfection with PAstV-2 and PAstV-5 in the same individual pigs.

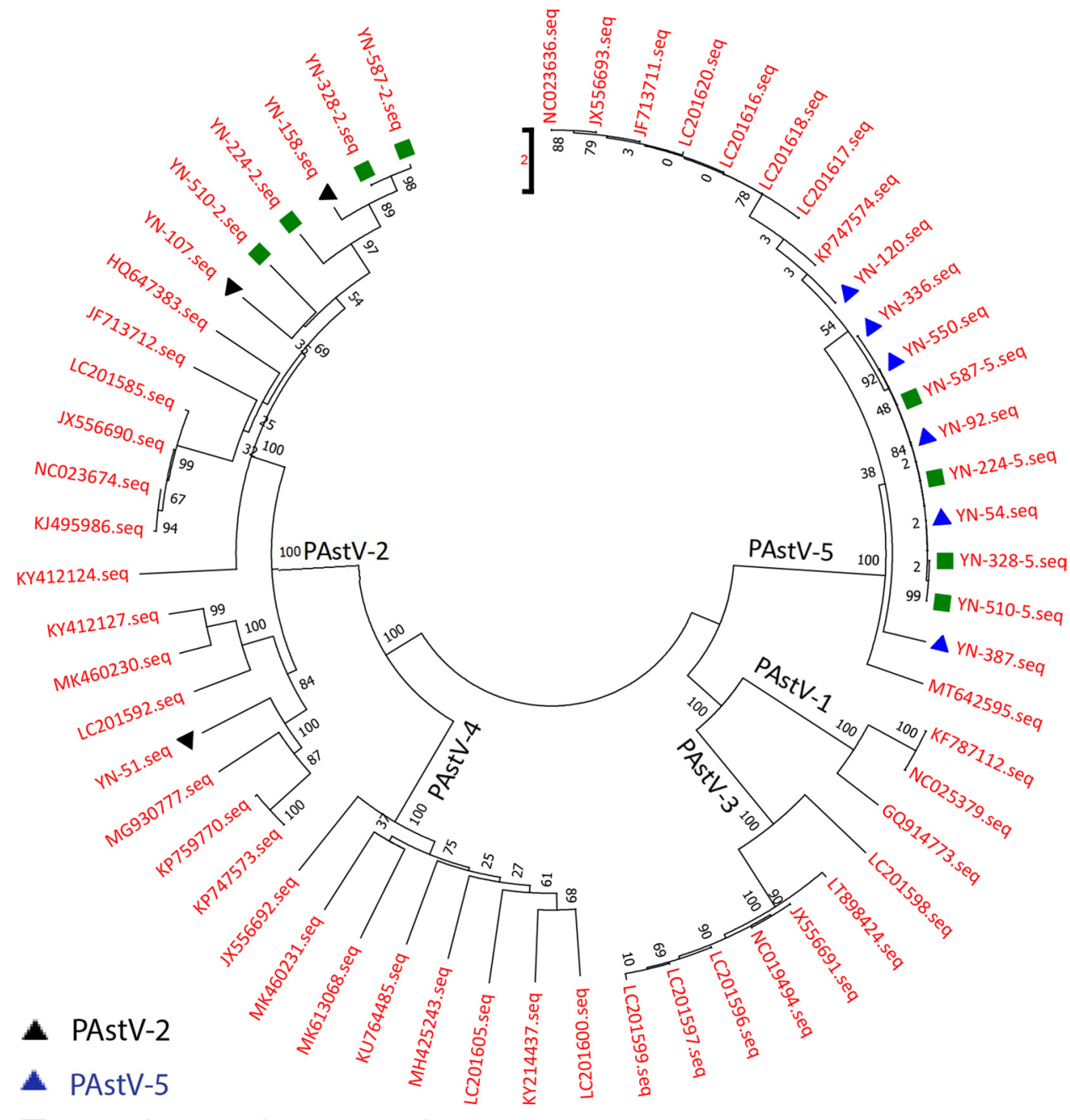

Co-infection of PAstV-2 and PAstV-5 divided into five groups in the phylogenetic tree, representing the five distinct genotypes from PAstV-1 to PAstV-5. Seven PAstV isolates from this study and 13 reference strains from five countries were placed into the PAstV-2 group and divided into two clades (Fig. 2). Five of the six PAstV-2 isolates from this study and seven PAstV-2 reference strains formed one clade in the PAstV-2 group, which shared 60.4-73.5\% nucleotide sequence identity (Fig. 3A). PAstV-2 strain YN-51 and six PAstV-2 reference strains shared 59.6-73.1\% nucleotide sequence identity and formed the other clade in the PAstV-2 group (Fig. 3A). Ten PAstV-5 ORF2 gene sequences from this study clustered with the PAstV-5 group (Fig. 2) and had 70.4-100\% nucleotide sequence identity (Fig. 3B).

The nucleotide sequence identity of the seventeen PAstV ORF2 genes identified in this study ranged from 59.5 to $100 \%$, indicating a wide variation at the nucleotide level, as was reported previously for RdRp gene sequences [49]. Notably, the ORF2 sequence of YN-387 showed relatively low sequence similarity (71.7-77.9\% identity, Fig. 3B, indicated by a green square) to the other 19 PAstV-5 sequences, including the other nine PAstV-5 sequences from Yunnan, demonstrating the potential variability of the ORF2 region.

\section{Amino acid sequence comparisons of PAstV-2 and PAstV-5 isolates from Yunnan}

The seven PAstV-2 capsid protein sequences from this study shared $64.8-96.3 \%$ amino acid sequence identity (Fig. 4A), while the 10 newly identified PAstV-5 strains had $85.9-100 \%$ identity (Fig. 4B), demonstrating a wide variation at the amino acid level. Further sequence alignment showed that YN-107 had a unique deletion of 37 amino acids (aa 1674-1710) when compared with the other PAstV-2 strains (indicated by a blue square in Fig. 5A). On the other hand, all of thePAstV-2 capsid protein sequences except for YN-51 contained an insertion of six amino acids (aa 1763-1768, indicated by a blue square in Fig. 5B). Furthermore, four of the seven PAstV-2 capsid protein 


\begin{tabular}{|c|c|c|c|c|c|c|c|c|c|c|c|c|c|c|c|c|c|c|c|c|c|}
\hline \multirow{2}{*}{ A } & \multicolumn{21}{|c|}{ Percent Identity } \\
\hline & 1 & 2 & 3 & 4 & 5 & 6 & 7 & 8 & 9 & 10 & 11 & 12 & 13 & 14 & 15 & 16 & 17 & 18 & 19 & 20 & \\
\hline 1 & & 85.6 & 79.0 & 85.6 & 67.7 & 73.0 & 70.6 & 71.2 & 62.8 & 70.0 & 73.6 & 70.1 & 85.5 & 61.7 & 65.9 & 66.8 & 64.0 & 67.4 & 70.2 & 66.6 & 1 \\
\hline 2 & 16.2 & & 83.3 & 90.0 & 67.2 & 71.7 & 71.1 & 71.3 & 62.5 & 70.9 & 71.6 & 69.3 & 83.5 & 61.5 & 66.1 & 66.1 & 63.5 & 66.8 & 70.9 & 66.5 & 2 \\
\hline 3 & 24.9 & 19.1 & & 88.7 & 67.2 & 72.1 & 71.2 & 70.9 & 62.3 & 70.2 & 71.5 & 69.2 & 78.1 & 61.1 & 69.0 & 66.1 & 63.5 & 72.0 & 67.5 & 73.5 & 3 \\
\hline 4 & 16.2 & 10.9 & 12.3 & & 67.4 & 71.9 & 71.1 & 71.1 & 62.5 & 70.6 & 71.6 & 69.3 & 83.5 & 61.4 & 66.6 & 66.5 & 63.3 & 66.8 & 70.5 & 67.2 & 4 \\
\hline 5 & 42.5 & 43.6 & 43.6 & 43.2 & & 70.0 & 67.0 & 61.0 & 60.4 & 65.3 & 67.3 & 62.8 & 67.4 & 63.2 & 61.3 & 60.4 & 63.5 & 62.6 & 63.3 & 62.1 & 5 \\
\hline 6 & 33.6 & 35.7 & 35.2 & 35.5 & 38.7 & & 71.1 & 66.7 & 62.9 & 70.2 & 70.5 & 68.0 & 72.1 & 64.8 & 63.8 & 64.2 & 62.3 & 64.9 & 66.0 & 64.6 & 6 \\
\hline 7 & 37.5 & 36.8 & 36.8 & 36.8 & 43.9 & 36.9 & & 69.5 & 65.9 & 78.6 & 85.0 & 71.2 & 70.5 & 72.9 & 61.4 & 59.2 & 60.5 & 60.6 & 61.1 & 59.4 & 7 \\
\hline 8 & 36.6 & 36.5 & 37.2 & 36.8 & 55.3 & 44.3 & 39.4 & & 80.2 & 69.9 & 69.7 & 72.8 & 70.2 & 59.6 & 56.5 & 58.1 & 57.4 & 58.3 & 57.4 & 57.9 & 8 \\
\hline 9 & 51.5 & 52.3 & 52.6 & 52.4 & 56.8 & 51.4 & 45.7 & 23.3 & & 66.9 & 65.6 & 72.4 & 62.6 & 61.1 & 56.4 & 57.1 & 56.1 & 57.9 & 57.3 & 57.4 & 9 \\
\hline 10 & 38.7 & 37.3 & 38.4 & 37.7 & 46.9 & 38.3 & 25.5 & 38.8 & 44.1 & & 75.3 & 71.9 & 70.0 & 66.9 & 60.5 & 61.1 & 62.1 & 60.9 & 61.4 & 60.1 & 10 \\
\hline 11 & 32.7 & 35.9 & 36.2 & 36.1 & 43.2 & 37.9 & 16.9 & 39.0 & 46.4 & 30.3 & & 73.0 & 75.0 & 73.1 & 61.1 & 59.0 & 60.4 & 60.6 & 60.8 & 59.6 & 11 \\
\hline 12 & 38.4 & 39.8 & 40.0 & 39.8 & 51.6 & 41.8 & 36.6 & 34.1 & 34.9 & 35.5 & 33.8 & & 70.1 & 60.9 & 55.3 & 56.1 & 55.7 & 55.9 & 57.4 & 55.2 & 12 \\
\hline 13 & 16.4 & 18.9 & 26.2 & 19.0 & 43.1 & 35.2 & 37.9 & 38.3 & 52.1 & 38.6 & 30.9 & 38.4 & & 61.7 & 67.4 & 67.0 & 64.3 & 67.5 & 71.1 & 67.3 & 13 \\
\hline 14 & 53.8 & 54.4 & 55.4 & 54.7 & 50.9 & 47.9 & 33.9 & 58.5 & 55.3 & 44.0 & 33.6 & 55.5 & 54.0 & & 61.2 & 59.6 & 60.5 & 60.2 & 59.6 & 59.5 & 14 \\
\hline 15 & 45.7 & 45.3 & 40.2 & 44.5 & 55.0 & 49.9 & 54.6 & 65.5 & 65.9 & 56.4 & 55.2 & 68.5 & 43.1 & 55.0 & & 70.4 & 67.8 & 72.5 & 70.6 & 72.6 & 15 \\
\hline 16 & 44.0 & 45.3 & 45.4 & 44.8 & 57.0 & 49.0 & 59.3 & 61.7 & 64.3 & 55.1 & 59.8 & 66.4 & 43.8 & 58.4 & 37.8 & & 86.5 & 88.2 & 69.4 & 87.6 & 16 \\
\hline 17 & 49.4 & 50.5 & 50.5 & 50.9 & 50.5 & 52.7 & 56.4 & 63.5 & 66.8 & 53.1 & 56.5 & 67.4 & 48.9 & 56.6 & 42.5 & 14.9 & & 86.1 & 67.7 & 85.2 & 17 \\
\hline 18 & 43.0 & 44.2 & 35.3 & 44.2 & 52.4 & 47.7 & 56.1 & 61.4 & 62.3 & 55.6 & 56.2 & 66.9 & 42.9 & 57.1 & 34.6 & 12.9 & 15.4 & & 69.6 & 93.6 & 18 \\
\hline 19 & 38.1 & 37.0 & 42.9 & 37.7 & 50.9 & 45.6 & 55.0 & 63.2 & 63.7 & 54.5 & 55.7 & 63.1 & 36.7 & 58.4 & 37.6 & 39.6 & 42.5 & 39.2 & & 70.0 & 19 \\
\hline \multirow[t]{2}{*}{20} & 44.3 & 44.5 & 32.8 & 43.4 & 53.4 & 48.0 & 58.7 & 62.1 & 63.5 & 57.2 & 58.3 & 68.5 & 43.2 & 58.6 & 34.3 & 13.5 & 16.4 & 6.7 & 38.6 & & 20 \\
\hline & 1 & 2 & 3 & 4 & 5 & 6 & 7 & 8 & 9 & 10 & 11 & 12 & 13 & 14 & 15 & 16 & 17 & 18 & 19 & 20 & \\
\hline
\end{tabular}

JX556690.seq KJ495986.seq JF713712.seq NC023674.seq KY412124.seq HQ647383.seq KP747573.seq MK460230.seq KY412127.seq MG930777.seq KP759770.seq LC201592.seq LC201585.seq

YN-51.seq

$\mathrm{YN}-107$.seq YN-158.seq

YN-224-2.seq YN-328-2.seq YN-510-2.seq YN-587-2.seq

\begin{tabular}{|c|c|c|c|c|c|c|c|c|c|c|c|c|c|c|c|c|c|c|c|c|c|c|}
\hline \multirow[b]{2}{*}{ B } & \multicolumn{22}{|c|}{ Percent Identity } \\
\hline & 1 & 2 & 3 & 4 & 5 & 6 & 7 & 8 & 9 & 10 & 11 & 12 & 13 & 14 & 15 & 16 & 17 & 18 & 19 & 20 & & \\
\hline 1 & & 84.3 & 84.8 & 84.8 & 88.9 & 84.3 & 85.1 & 84.5 & 72.3 & 85.2 & 71.7 & 71.9 & 71.2 & 71.9 & 70.6 & 71.9 & 72.7 & 71.0 & 72.3 & 71.9 & 1 & MT642595.seq \\
\hline 2 & 18.0 & & 88.6 & 88.6 & 84.7 & 100.0 & 88.7 & 96.7 & 75.7 & 88.7 & 81.7 & 81.7 & 81.5 & 81.7 & 80.5 & 81.7 & 72.4 & 80.8 & 82.0 & 81.8 & 2 & NC023636.seq \\
\hline 3 & 17.4 & 12.7 & & 90.5 & 85.1 & 88.6 & 94.1 & 88.7 & 76.2 & 94.1 & 82.0 & 82.2 & 82.3 & 82.3 & 81.4 & 82.4 & 72.4 & 81.1 & 82.4 & 82.3 & 3 & LC201616.seq \\
\hline 4 & 17.3 & 12.7 & 10.4 & & 85.1 & 88.6 & 91.5 & 88.5 & 76.2 & 91.5 & 81.9 & 81.5 & 82.0 & 81.6 & 80.1 & 81.5 & 71.8 & 80.0 & 82.0 & 81.6 & 4 & LC201620.seq \\
\hline 5 & 12.4 & 17.4 & 17.0 & 17.0 & & 84.7 & 85.7 & 84.6 & 72.6 & 85.7 & 71.2 & 71.1 & 71.0 & 71.2 & 70.4 & 71.4 & 71.7 & 70.5 & 71.5 & 71.3 & 5 & LC201619.seq \\
\hline 6 & 18.0 & 0.0 & 12.7 & 12.7 & 17.4 & & 88.7 & 96.7 & 75.7 & 88.7 & 81.7 & 81.7 & 81.5 & 81.7 & 80.5 & 81.7 & 72.4 & 80.8 & 82.0 & 81.8 & 6 & JX556693.seq \\
\hline 7 & 16.9 & 12.5 & 6.2 & 9.2 & 16.1 & 12.5 & & 89.0 & 76.2 & 100.0 & 82.0 & 81.9 & 82.3 & 82.0 & 80.9 & 81.8 & 72.6 & 80.8 & 82.3 & 82.0 & 7 & LC201618.seq \\
\hline 8 & 17.7 & 3.4 & 12.5 & 12.8 & 17.6 & 3.4 & 12.2 & & 75.7 & 89.0 & 81.7 & 81.8 & 82.4 & 81.8 & 80.4 & 81.8 & 72.2 & 81.0 & 82.2 & 82.0 & 8 & JF713711.seq \\
\hline 9 & 35.0 & 29.8 & 29.0 & 29.1 & 34.5 & 29.8 & 29.0 & 29.8 & & 76.2 & 93.3 & 93.4 & 96.4 & 93.5 & 92.4 & 93.7 & 75.6 & 92.3 & 93.3 & 93.1 & 9 & KP747574.seq \\
\hline 10 & 16.8 & 12.5 & 6.2 & 9.2 & 16.1 & 12.5 & 0.0 & 12.2 & 29.0 & & 82.0 & 81.9 & 82.3 & 82.0 & 80.9 & 81.8 & 72.6 & 80.8 & 82.3 & 82.0 & 10 & LC201617.seq \\
\hline 11 & 36.0 & 21.5 & 21.0 & 21.2 & 36.8 & 21.5 & 20.9 & 21.5 & 7.1 & 20.9 & & 99.0 & 92.0 & 99.0 & 93.4 & 98.3 & 77.9 & 93.4 & 98.1 & 97.9 & 11 & YN-54.seq \\
\hline 12 & 35.7 & 21.5 & 20.7 & 21.7 & 37.0 & 21.5 & 21.1 & 21.3 & 7.0 & 21.1 & 1.1 & & 92.0 & 100.0 & 93.6 & 98.3 & 77.8 & 93.5 & 98.2 & 98.0 & 12 & YN-92.seq \\
\hline 13 & 36.7 & 21.8 & 20.6 & 21.1 & 37.1 & 21.8 & 20.6 & 20.5 & 3.7 & 20.6 & 8.6 & 8.6 & & 92.1 & 91.4 & 92.4 & 75.0 & 91.4 & 92.1 & 91.9 & 13 & YN-120.seq \\
\hline 14 & 35.6 & 21.4 & 20.6 & 21.6 & 36.9 & 21.4 & 21.1 & 21.3 & 6.9 & 21.1 & 1.0 & 0.0 & 8.5 & & 93.6 & 98.4 & 77.9 & 93.5 & 98.3 & 98.1 & 14 & YN-224-5.seq \\
\hline 15 & 37.8 & 23.1 & 21.9 & 23.7 & 38.3 & 23.1 & 22.6 & 23.3 & 8.1 & 22.6 & 7.0 & 6.8 & 9.3 & 6.8 & & 93.8 & 75.6 & 98.5 & 93.1 & 92.8 & 15 & YN-328-5.seq \\
\hline 16 & 35.5 & 21.5 & 20.5 & 21.7 & 36.6 & 21.5 & 21.3 & 21.3 & 6.7 & 21.3 & 1.7 & 1.7 & 8.1 & 1.7 & 6.6 & & 77.8 & 93.8 & 98.1 & 97.9 & 16 & YN-336.seq \\
\hline 17 & 34.2 & 34.7 & 34.7 & 35.8 & 35.9 & 34.7 & 34.5 & 35.0 & 29.7 & 34.5 & 26.4 & 26.4 & 30.7 & 26.4 & 29.8 & 26.4 & & 75.9 & 78.0 & 77.8 & 17 & YN-387.seq \\
\hline 18 & 37.1 & 22.7 & 22.2 & 23.8 & 38.0 & 22.7 & 22.8 & 22.5 & 8.2 & 22.8 & 7.0 & 6.9 & 9.3 & 6.9 & 1.6 & 6.6 & 29.3 & & 93.2 & 92.9 & 18 & YN-510-5.seq \\
\hline 19 & 34.9 & 21.0 & 20.4 & 21.1 & 36.3 & 21.0 & 20.6 & 20.8 & 7.1 & 20.6 & 1.9 & 1.8 & 8.5 & 1.8 & 7.4 & 1.9 & 26.1 & 7.3 & & 99.4 & 19 & YN-550.seq \\
\hline \multirow[t]{2}{*}{20} & 35.5 & 21.3 & 20.6 & 21.5 & 36.7 & 21.3 & 21.1 & 21.1 & 7.3 & 21.1 & 2.2 & 2.0 & 8.8 & 2.0 & 7.7 & 2.2 & 26.4 & 7.6 & 0.6 & & 20 & YN-587-5.seq \\
\hline & 1 & 2 & 3 & 4 & 5 & 6 & 7 & 8 & 9 & 10 & 11 & 12 & 13 & 14 & 15 & 16 & 17 & 18 & 19 & 20 & & \\
\hline
\end{tabular}

Fig. 3 Nucleotide acid sequence comparison of the 17 Yunnan PAstV ORF2 sequences with 23 selected reference sequences. DNAStar 6.0 software was used with default parameters to compare the ORF2 gene sequences between the 23 selected reference sequences (Supplementary Table S1) and the 17 sequences obtained in this study (Supplementary Table S2). The seven PAstV-2 (A) and

sequences (YN-158, YN-224, YN-328, and YN-587) had an insertion of nine amino acids (aa 2054-2062, NLDLDPGD, indicated by a blue square in Fig. 5C), while YN-51 had an insertion of three amino acids (LED, Fig. 5C), and no insertion was present at the same site for YN-510. When compared with the 10 selected reference sequences from China and other countries (the representative region ten PAstV-5 (B) ORF2 gene sequences from this study (both indicated by a red square) shared 59.5\%-93.6\% and 75\%-100\% nucleotide sequence identity, respectively. However, YN-387 (PAstV-5 genotype, B) diverged from the other PAstV-5 ORF2 gene sequences, with sequence identity between $70 \%$ and $80 \%$ (indicated by a green square).

between amino site 1700 and 1787 is shown in Fig. 6), many point mutations were observed in the $10 \mathrm{PAstV}-5$ capsid sequences identified in this study that were also present in the reference sequence KP747574 from Beijing (indicated by a blue square) [52], but not in the reference sequence MT642595 from a CSFV-infected specimen from 


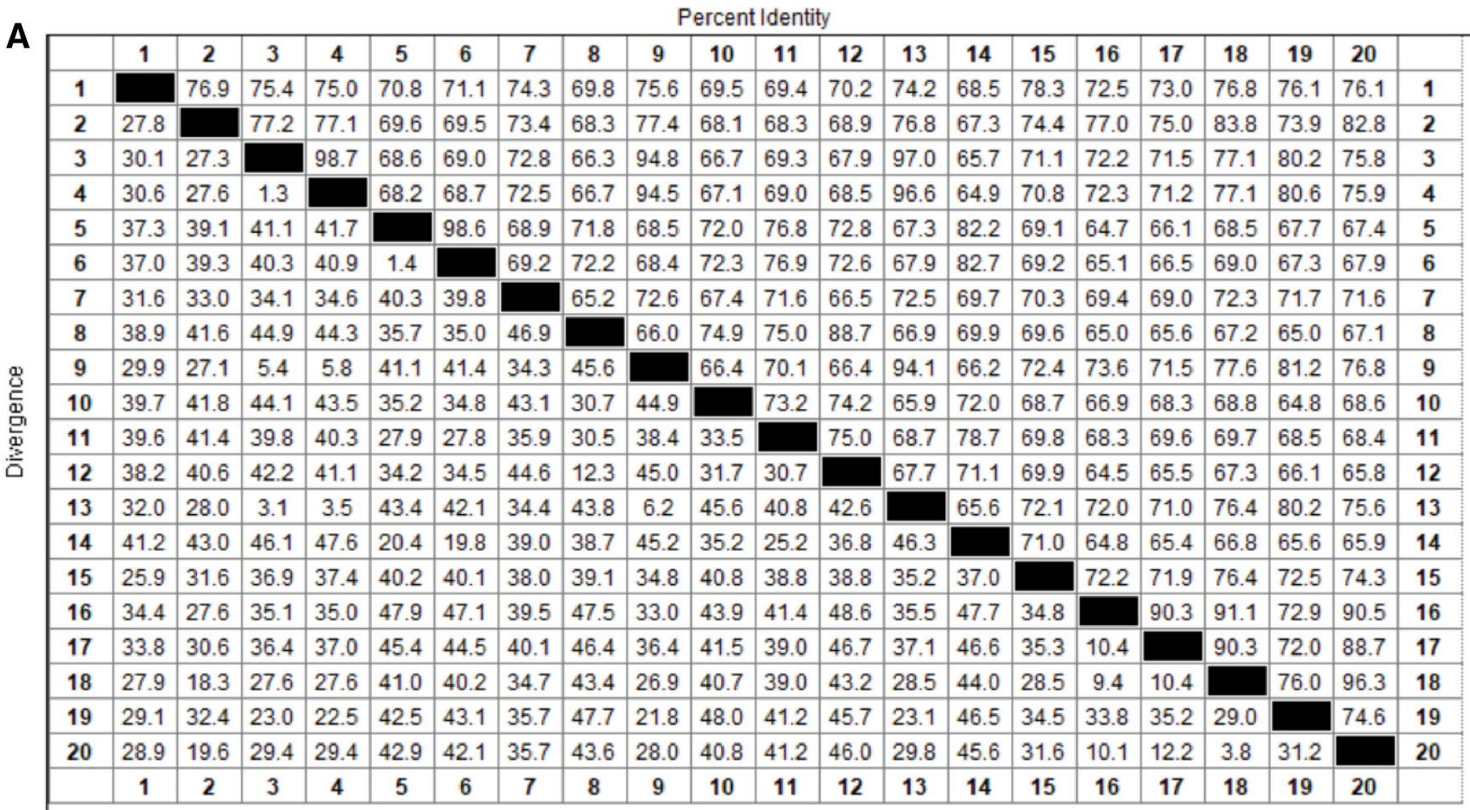

HQ647383.seq JF713712.seq JX556690.seq KJ495986.seq KP747573.seq KP759770.seq KY 412124 .seq KY412127.seq LC201585.seq LC201592.seq MG930777.seq MK460230.seq NC023674.seq YN-51.seq YN-107.seq YN-158.seq YN-224-2.seq YN-328-2.seq YN-510-2.seq YN-587-2.seq

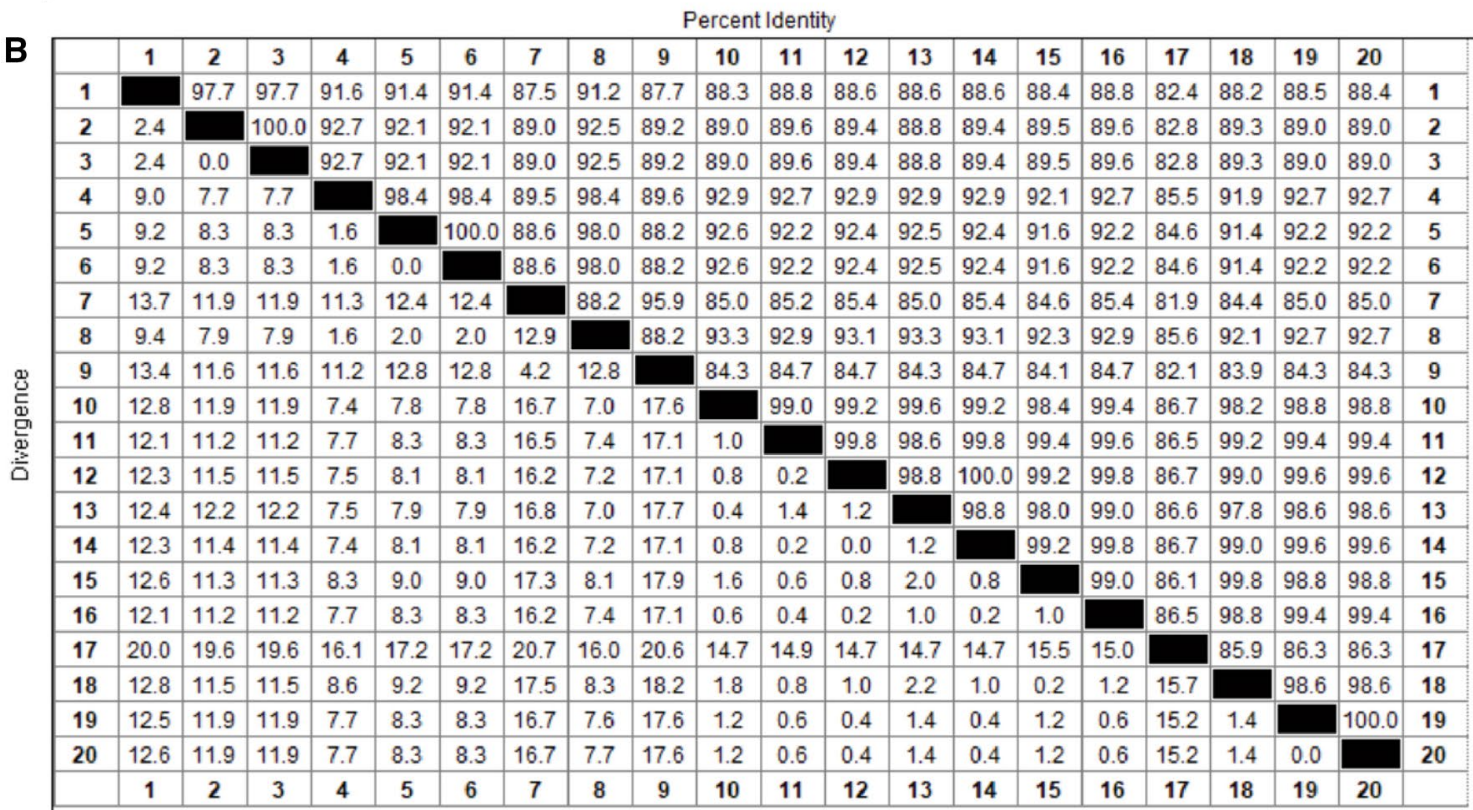

JF713711.seq JX556693.seq NC023636.seq LC201616.seq LC201617.seq LC201618.seq LC201619.seq LC201620.seq MT642595.seq KP747574.seq YN-54.seq YN-92.seq $\mathrm{YN}-120$.seq YN-224-5.seq YN-328-5.seq YN-336.seq YN-387.seq YN-510-5.seq YN-550.seq YN-587-5.seq
Fig. 4 Amino acid sequence comparison of the 17 Yunnan PAstV capsid proteins with the 23 selected reference sequences. DNAStar 6.0 software with default parameters was used to compare the capsid protein sequences between the 23 selected reference sequences (Supplementary Table S1) and the 17 sequences obtained in this study
(Supplementary Table S2). The seven PAstV-2 (A) and 10 PAstV-5 (B) capsid protein sequences from this study (both indicated by a red square) shared $64.8 \%-90.3 \%$ and $86.1 \%-100 \%$ amino acid sequence identity, respectively.
Anhui province, China [42]. This observation may imply a separate domestic origin of Yunnan PAstV-5 strains. Determining whether the indels (insertions and deletions) and mutations are associated with differences in the infectivity and pathogenicity of PAstV-2 and PAstV-5, and if so, investigating the underlying mechanisms, would be worth further functional studies.

\section{Discussion}

It is becoming increasingly recognized that pigs harbor a wide spectrum of viruses with long-term persistence, serving as reservoirs for numerous human zoonotic diseases. Porcine astrovirus (PAstV) is distributed globally and represented by at least five distinct genotypes (PAstV-1 to PAstV-5). PAstV can cause diarrhea, vomiting, and even 
A

\begin{tabular}{|l|}
\hline 1. HQ647383.seq \\
\hline 2. JF713712.seq \\
\hline 3. JX556690.seq \\
\hline 4. KJ495986.seq \\
\hline 5. KP747573.seq \\
\hline 6. KP759770.seq \\
\hline 7. KY412124.seq \\
\hline 8. KY412127.seq \\
\hline 9. LC201585.seq \\
\hline 10. LC201592.seq \\
\hline 11. MG930777.seq \\
\hline 12. MK460230.seq \\
\hline 13. NC023674.seq \\
\hline 14. YN-51.seq \\
\hline 15. YN-107.seq \\
\hline 16. YN-158.seq \\
\hline 17. YN-224-2.seq \\
\hline 18. YN-328-2.seq \\
\hline 19. YN-510-2.seq \\
\hline 20. YN-587-2.seq \\
\hline
\end{tabular}

B

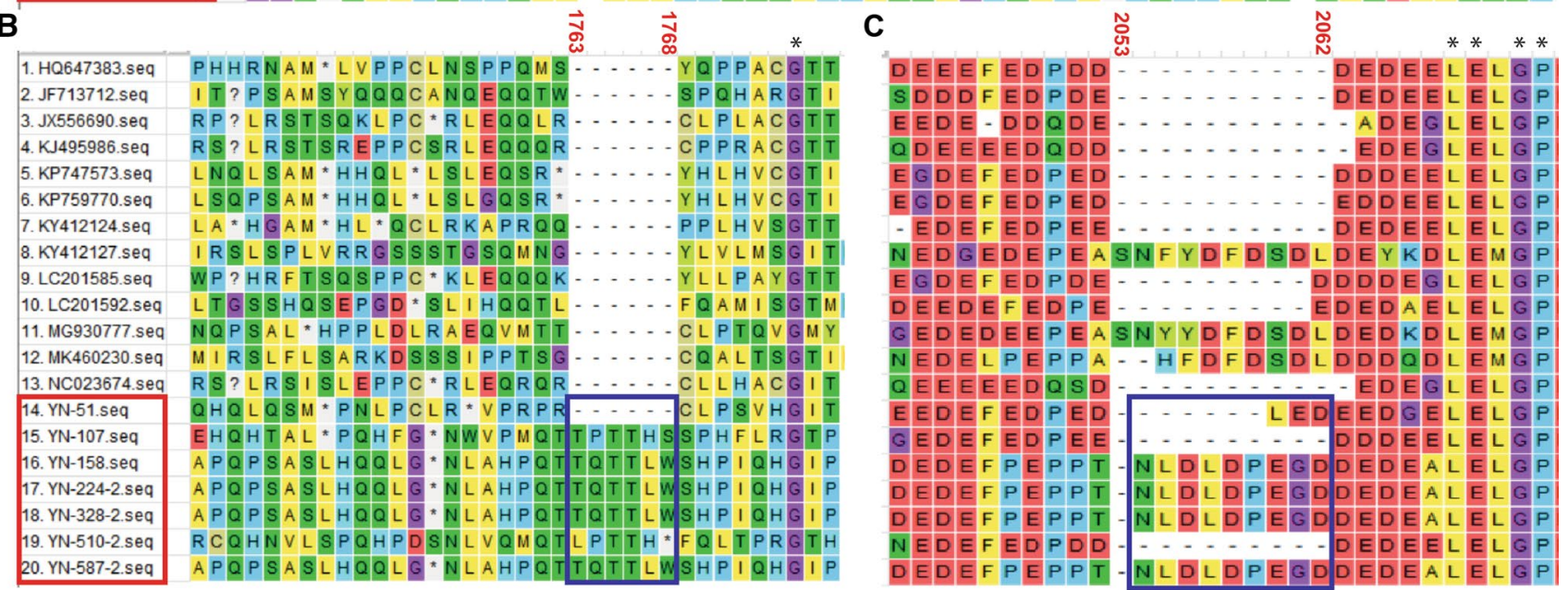

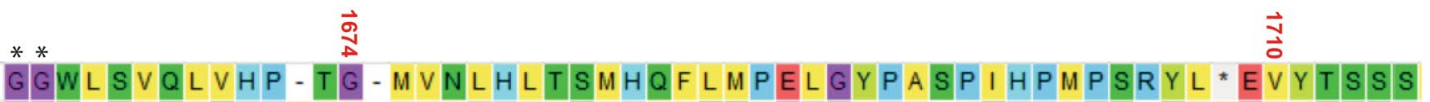
GGS * SVQLTRPFAA - MAAPRL I SMHLFQTLGPTLHA *LTRPAST-SLLGVFTSSR GGLSNVQLEHQCAV - VR - QHSTSTPQSLMLGRTLPALLTK - I ST - SMLAGSTSSK GGL *NVQLEHQFAV-VR-PHLTSMPQFLMPGRTLPALLTK-RST-SMWAGFTFSR GGSLKGSPEPPRG - - LALSNLPSTHRSTMRVPMFPAYHHP* MLALLALASSTSSR GGSTKGSPEPP * G- - L A L SNLPSTHRLTMRVPMFPAYHHP *TLALSALASSTSSR GG * *NGLLVPLFVV-TGTWLLISTHPSQMRGRTCLA*MVSNQST - *Q*EASIFNK GGSSSASREHRPV - A A LRPSRSILL *MMLGQVCLA*LRQQIRGVSTSGSYTSSR GGLSSVQLAHQCAV - VR - QHSTSMPQFLMPGRIRPASLTS - RST - SMWAGSTSNR GGS *NGLQEHRPAP - VEWRPLRYTLPSMMLEQVCPV *LRQSIKEASVLGNFISSK GGSSRRLGGPPAG - VAPRGS * FMPQSTMPGPASHVCQQQPMQTLLMLVSSTSSR GGS *NALLVHQLA- - AEWRLSRSILPLTMLEQMFPVLLTPPISAQ *ASGSCISSR GGSSNVQLEHQCAV - VR - QHLTSTPQFPTLDRTPHASQTK-RST-ST*AGFTSSR GGS * RGLAELLFG- - LEQNGS ${ }^{2} M H P$ * TMPEPMYHAFHQNKMPPLWVSASSTSSR GGWS N A P P TL PSVLGGSSRERQVRLFA Q TMVRYALISTTR SQMHGPIHHACCSRG *KLPSR *VVSTSSR GGW * SARRVPRSA - - LERCALTSTLPSQMLGQTRLASFSKASPNLFRLVVSTSSR GGS *NAPPTHPFAA - TGTSHLTSTHQFPMPVPTCHVSLTRLLST-SQWVASTSSR GGWSNVQLVLQCVK - ALRPLTSTRQFPMRVQVYPA * STVQSKLPSMLVVYTSNR GGS * SVLPTRPSAA-MVAPHSTSMHPFQTLGPTLHA *PTRPAST - SLSEVFTSNR

Fig. 5 Comparison of the deduced amino acid sequences of seven PAstV-2 capsid proteins with those of 13 selected reference sequences. (A) The capsid protein sequence of YN-107 displayed a consecutive deletion of 37 aa (between aa 1674 and 1710), as indicated by a blue square. (B) Except for YN-51, the PAstV-2 capsid protein sequences contained an insertion of 6 aa at the $\mathrm{N}$-terminus (between aa 1763 and 1768), as indicated by a blue square, thus dif-

death in piglets, resulting in great economic losses in the pig industry, especially when present in mixed infections with other porcine pathogens. More importantly, previous studies have suggested that PAstV-1, PAstV-2, PAstV-3, and PAstV-5 may have been transmitted across host species $[9,53]$. For example, the PAstV-2 isolate HQ647383, a reference sequence used in this study, very likely underwent two recombination events between porcine and deer AstVs [41], and possible recombination between porcine and human AstVs was also observed in Colombia [21]. As there have been no studies on PAstV epidemiology in fering from the selected reference sequences. (C) Insertion of NLDLDPEGD at aa position 2053 in YN-158, YN-224-2, YN-328-2, and YN-587-2, insertion of LED at the same position in YN-51, and no insertion in YN-107 and YN-510-2 (indicated by a blue square). The seven PAstV-2 capsid protein sequences from this study are indicated by red squares. The asterisks $(*)$ indicate positions where the amino acid is identical in all 20 sequences.
Yunnan, we investigated PAstV epidemiology in Yunnan in this study.

In the present study, 489 fecal samples were collected from four different pig populations across Yunnan's 16 prefectures/cities between April and August of 2020 to screen for all five genotypes of PAstV. However, only PAstV-2 and PAstV-5 were detected. There are two possible reasons for this: either the other three genotypes were not present in the collected samples or the amount of virus was below the detection limit of the assay. Further optimization is needed to investigate this question. 


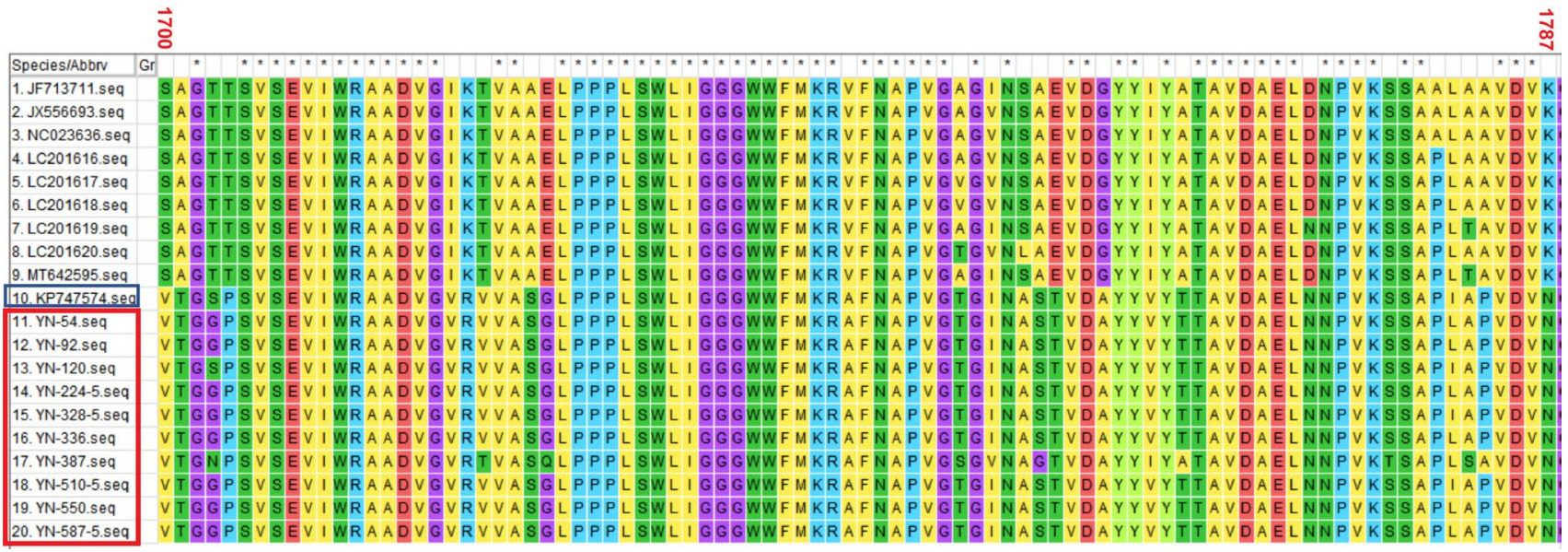

Fig. 6 Comparison of the 10 deduced amino acid sequences of PAstV-5 capsid proteins with 10 selected reference sequences. The 10 PAstV-5 capsid protein sequences from this study (indicated by a red square) shared a high degree of similarity with each other $(81.9 \%$ $100 \%$ identity) but diverged from the reference sequences, with various individual amino acid substitutions. However, most of the

In this study, fecal samples were collected during summer and autumn and approximately one-third of the 489 samples were diarrheic. We found $107 \mathrm{PAstV}-2$-positive samples (21.9\%) and 92 PAstV-5-positive samples (18.8\%), representing an overall PAstV infection rate of $39.9 \%$, which is much higher than the positive rate of PAstVs in feces samples from Sichuan province during the winter of 2014 $(17.5 \%, 21 / 120)$ [39], and in those from an epidemiological investigation of diarrheic piglets from 17 provinces or municipalities in China from 2015 to 2018 (16.4\%, 89/543) [46]. Diarrhea is more prevalent in piglets throughout the winter and spring when pigs are more vulnerable to infections with diarrhea-inducing pathogens. Furthermore, piglets, sows, and finisher pigs were included in the sampling in this study in order to have a wide range of pigs of all ages and both sexes. As a result, it is reasonable to speculate that the PAstV infection rate would have been much higher if the samples had been collected in winter and spring or if the samples had been collected only from piglets with diarrhea. The presence of PAstV-2 or PAstV-5 in all Yunnan prefectures/cities and in all of the pig populations with rather high infection rates indicates a high prevalence of $\mathrm{PAstV}$ infection in Yunnan. Furthermore, a slightly higher PAstV-2 positivity rate was observed in clinically diarrheic pigs (46/151, $59.0 \%)$ than in non-diarrheic pigs $(61 / 338,52.1 \%)$, which was not the case for PAstV-5 infection $(46.2 \%$ in clinically diarrheic pigs vs. $47.6 \%$ in non-diarrheic pigs). Moreover, PAstV-2 was more prevalent than PAstV-5 (54.3\% vs. $48.1 \%$ in suckling piglets, $56.3 \%$ vs. $46.9 \%$ in weaned piglets, and $57.7 \%$ vs. $42.3 \%$ in finisher pigs), with no difference in sows (50\% vs. 50\%). In addition to the high prevalence of PAstV mutations in the 10 PAstV-5 sequences from this study were nearly identical to those in the reference sequence KP747574 (from Beijing, China, indicated by a blue square), as shown in the representative sequence alignment presented here, showing amino acids 1700 and 1787 . The asterisks (*) indicate positions where the amino acid is identical in all 20 sequences.

infection, coinfections with $\mathrm{PAstV}$ and other porcine viruses such as PBoV, PoSaV, and PCV2 were also evident in this study, which is consistent with a previous study [40]. Coinfections may contribute to a high rate of diarrhea and mortality, particularly in piglets, and thus a further deterioration of the swine industry, which merits significant attention from the health and anti-epidemic departments in Yunnan.

Phylogenetic analysis revealed that PAstV-2 and PAstV-5 were predominant in Yunnan between April and August of 2020, which is consistent with previous studies in Guangxi [35], Hunan [38, 39], Sichuan [40], and Shandong and Jiangxi [49]. While various insertions were observed in the $\mathrm{PAstV}-2$ sequences determined in this study, one PAstV-2 sequence (YN-107) was found to harbor a unique deletion of 37 amino acids within the capsid protein sequence, which, to our knowledge, is not present in any other PAstV-2 sequences in the NCBI database and thus worth further investigation. These findings expand our current understanding of the genetic characteristics of PAstV in Yunnan and provide valuable information for further PAstV studies. Altogether, the indels and mutations in the capsid proteins of the PAstV-2 and PAstV-5 genotypes from Yunnan may result in changes in viral immunogenicity and infectivity, potentially complicating vaccine development.

In conclusion, this study demonstrates for the first time that PAstV-2 and PastV-5 were prevalent in pig herds across all 16 prefectures/cities in Yunnan between April and August of 2020 , with infection rates of $21.9 \%$ and $18.8 \%$, respectively. Coinfections with PAstV-2 and PAstV-5 were detected in four pigs with diarrhea (YN-158, YN-224, YN-328, and YN-587), while single infections with PAstV-2 or PAstV-5 
were found in pigs with and without diarrhea, suggesting that double infections with PAstV-2 and PAstV-5 might increase the severity of disease. Coinfections with PAstV and multiple porcine viruses are prevalent and may be more common with PBoV and PoSaV. The high genetic variability of PAstV and its adaptation to various hosts suggest the possibility of cross-species transmission with enhanced pathogenicity. The findings of this study will offer a foundation for future prevention and control of PAstV infection in Yunnan province through the implementation of effective vaccination strategies.

Supplementary Information The online version contains supplementary material available at https://doi.org/10.1007/s00705-021-05311-8.

Acknowledgements This work was supported by the National Natural Science Foundation of China (Grant no. 31960701) and by the Program for Innovative Research Team (in Science and Technology) in the University of Yunnan Province (IRTSTYN). The funders had no role in study design, data collection and interpretation, or the decision to submit the work for publication. We are grateful to the colleagues who work on the pig farms and collected the fecal samples.

\section{Declarations}

Conflict of interest The author declares that they have no conflict of interest.

\section{References}

1. Guo L, Xu X, Song J, Wang W, Wang J, Hung T (2010) Molecular characterization of astrovirus infection in children with diarrhea in Beijing, 2005-2007. J Med Virol 82:415-423. https://doi.org/ 10.1002/jmv. 21729

2. Li R, Zhang R, Tan P, Han Y, Chen Y, Wang Z, Han D, Zhang J, Xie J, Zhang R, Li J (2021) Quality evaluation of molecular diagnostic tests for astrovirus, sapovirus and poliovirus: a multicenter study. Clin Chim Acta 512:172-178. https://doi.org/10.1016/j.cca. 2020.11.006

3. Seltmann A, Corman VM, Rasche A, Drosten C, Czirjak GA, Bernard H, Struebig MJ, Voigt CC (2017) Seasonal Fluctuations of Astrovirus, But Not Coronavirus Shedding in Bats Inhabiting Human-Modified Tropical Forests. EcoHealth 14:272-284. https://doi.org/10.1007/s10393-017-1245-x

4. Monroe SS, Jiang B, Stine SE, Koopmans M, Glass RI (1993) Subgenomic RNA sequence of human astrovirus supports classification of Astroviridae as a new family of RNA viruses. J Virol 67:3611-3614. https://doi.org/10.1128/JVI.67.6.3611-3614.1993

5. Mendez E, Fernandez-Luna T, Lopez S, Mendez-Toss M, Arias CF (2002) Proteolytic processing of a serotype 8 human astrovirus ORF2 polyprotein. J Virol 76:7996-8002. https://doi.org/10.1128/ jvi.76.16.7996-8002.2002

6. Wang QH, Kakizawa J, Wen LY, Shimizu M, Nishio O, Fang ZY, Ushijima H (2001) Genetic analysis of the capsid region of astroviruses. J Med Virol 64:245-255. https://doi.org/10.1002/ jmv.1043

7. Laurin MA, Dastor M, L'Homme Y (2011) Detection and genetic characterization of a novel pig astrovirus: relationship to other astroviruses. Arch Virol 156:2095-2099. https://doi.org/10.1007/ s00705-011-1088-7
8. Shan T, Li L, Simmonds P, Wang C, Moeser A, Delwart E (2011) The fecal virome of pigs on a high-density farm. J Virol 85:11697-11708. https://doi.org/10.1128/JVI.05217-11

9. Xiao CT, Gimenez-Lirola LG, Gerber PF, Jiang YH, Halbur PG, Opriessnig T (2013) Identification and characterization of novel porcine astroviruses (PAstVs) with high prevalence and frequent co-infection of individual pigs with multiple PAstV types. J Gen Virol 94:570-582. https://doi.org/10.1099/vir.0.048744-0

10. Bridger JC (1980) Detection by electron microscopy of caliciviruses, astroviruses and rotavirus-like particles in the faeces of piglets with diarrhoea. Vet Rec 107:532-533

11. Shimizu M, Shirai J, Narita M, Yamane T (1990) Cytopathic astrovirus isolated from porcine acute gastroenteritis in an established cell line derived from porcine embryonic kidney. J Clin Microbiol 28:201-206. https://doi.org/10.1128/jcm.28.2.201-206.1990

12. Amimo JO, Machuka EM, Abworo EO, Vlasova AN, Pelle R (2020) Whole genome sequence analysis of porcine astroviruses reveals novel genetically diverse strains circulating in East African smallholder pig farms. Viruses. https://doi.org/10.3390/v1211 1262

13. Arruda B, Arruda P, Hensch M, Chen Q, Zheng Y, Yang C, Gatto IRH, Ferreyra FM, Gauger P, Schwartz K, Bradner L, Harmon K, Hause B, Li G (2017) Porcine astrovirus type 3 in central nervous system of swine with polioencephalomyelitis. Emerg Infect Dis 23:2097-2100. https://doi.org/10.3201/eid2312.170703

14. Matias Ferreyra FS, Bradner LK, Burrough ER, Cooper VL, Derscheid RJ, Gauger PC, Harmon KM, Madson D, Pineyro PE, Schwartz KJ, Stevenson GW, Zeller MA, Arruda BL (2020) Polioencephalomyelitis in domestic swine associated with porcine astrovirus type 3. Vet Pathol 57:82-89. https://doi.org/10.1177/ 0300985819875741

15. Mor SK, Chander Y, Marthaler D, Patnayak DP, Goyal SM (2012) Detection and molecular characterization of Porcine astrovirus strains associated with swine diarrhea. J Vet Diagn Invest 24:1064-1067. https://doi.org/10.1177/1040638712458781

16. Opriessnig T, Xiao CT, Halbur PG (2020) Porcine astrovirus type 5-associated enteritis in pigs. J Comp Pathol 181:38-46. https:// doi.org/10.1016/j.jcpa.2020.09.014

17. Padmanabhan A, Hause BM (2016) Detection and characterization of a novel genotype of porcine astrovirus 4 from nasal swabs from pigs with acute respiratory disease. Arch Virol 161:25752579. https://doi.org/10.1007/s00705-016-2937-1

18. Rawal G, Ferreyra FM, Macedo NR, Bradner LK, Harmon KM, Allison G, Linhares DCL, Arruda BL (2020) Ecology of porcine astrovirus type 3 in a herd with associated neurologic disease. Viruses. https://doi.org/10.3390/v12090992

19. Rawal G, Ferreyra FM, Macedo NR, Bradner LK, Harmon KM, Mueller A, Allison G, Linhares DCL, Arruda BL (2019) Detection and cellular tropism of porcine astrovirus type 3 on breeding farms. Viruses. https://doi.org/10.3390/v11111051

20. Xiao CT, Halbur PG, Opriessnig T (2012) Complete genome sequence of a newly identified porcine astrovirus genotype 3 strain US-MO123. J Virol 86:13126. https://doi.org/10.1128/JVI. 02426-12

21. Ulloa JC, Gutierrez MF (2010) Genomic analysis of two ORF2 segments of new porcine astrovirus isolates and their close relationship with human astroviruses. Can J Microbiol 56:569-577. https://doi.org/10.1139/w10-042

22. Ulloa JC, Guzman F, Guerrero CA, Gutierrez MF (2012) Identification of two immunoreactive peptides useful for the detection of porcine astrovirus. Intervirology 55:311-317. https://doi.org/ $10.1159 / 000328277$

23. Brnic D, Prpic J, Keros T, Roic B, Staresina V, Jemersic L (2013) Porcine astrovirus viremia and high genetic variability in pigs on large holdings in Croatia. Infect Genet Evol 14:258-264. https:// doi.org/10.1016/j.meegid.2012.12.027 
24. Tassoni L, Zamperin G, Schiavon E, Vendramin V, Cavicchio L, Mion M, Tonon FT, Monne I, Beato MS (2019) First whole genome characterization of porcine astrovirus detected in swine faeces in Italy. Vet Ital 55:221-229. https://doi.org/10.12834/ VetIt.1873.9956.1

25. Van Borm S, Vanneste K, Fu Q, Maes D, Schoos A, Vallaey E, Vandenbussche F (2020) Increased viral read counts and metagenomic full genome characterization of porcine astrovirus 4 and Posavirus 1 in sows in a swine farm with unexplained neonatal piglet diarrhea. Virus Genes 56:696-704. https://doi.org/10.1007/ s11262-020-01791-Z

26. Indik S, Valicek L, Smid B, Dvorakova H, Rodak L (2006) Isolation and partial characterization of a novel porcine astrovirus. Vet Microbiol 117:276-283. https://doi.org/10.1016/j.vetmic.2006.06. 020

27. Reuter G, Nemes C, Boros A, Kapusinszky B, Delwart E, Pankovics P (2012) Astrovirus in wild boars (Sus scrofa) in Hungary. Arch Virol 157:1143-1147. https://doi.org/10.1007/ s00705-012-1272-4

28. Reuter G, Pankovics P, Boros A (2011) Identification of a novel astrovirus in a domestic pig in Hungary. Arch Virol 156:125-128. https://doi.org/10.1007/s00705-010-0827-5

29. Reuter G, Pankovics P, Delwart E, Boros A (2012) Identification of a novel astrovirus in domestic sheep in Hungary. Arch Virol 157:323-327. https://doi.org/10.1007/s00705-011-1151-4

30. Kattoor JJ, Malik YS, Saurabh S, Sircar S, Vinodhkumar OR, Bora DP, Dhama K, Ghosh S, Banyai K, Touil N, Abdel-Moneim AS, Vlasova AN, Kobayashi N, Singh RK (2019) First report and genetic characterization of porcine astroviruses of lineage 4 and 2 in diarrhoeic pigs in India. Transbound Emerg Dis 66:47-53. https://doi.org/10.1111/tbed.13058

31. Kumthip K, Khamrin P, Saikruang W, Kongkaew A, Vachirachewin R, Ushijima H, Maneekarn N (2018) Detection and genetic characterization of porcine astroviruses in piglets with and without diarrhea in Thailand. Arch Virol 163:1823-1829. https:// doi.org/10.1007/s00705-018-3806-x

32. Lee MH, Jeoung HY, Park HR, Lim JA, Song JY, An DJ (2013) Phylogenetic analysis of porcine astrovirus in domestic pigs and wild boars in South Korea. Virus Genes 46:175-181. https://doi. org/10.1007/s11262-012-0816-8

33. Lee S, Jang G, Lee C (2015) Complete genome sequence of a porcine astrovirus from South Korea. Arch Virol 160:1819-1821. https://doi.org/10.1007/s00705-015-2436-9

34. Shirai J, Shimizu M, Fukusho A (1985) Coronavirus-, calicivirus-, and astrovirus-like particles associated with acute porcine gastroenteritis. Nihon Juigaku Zasshi 47:1023-1026. https://doi.org/10. 1292/jvms1939.47.1023

35. Qin Y, Fang Q, Li X, Li F, Liu H, Wei Z, Ouyang K, Chen Y, Huang W (2019) Molecular epidemiology and viremia of porcine astrovirus in pigs from Guangxi province of China. BMC Vet Res 15:471. https://doi.org/10.1186/s12917-019-2217-x

36. Fang Q, Wang C, Liu H, Wu Q, Liang S, Cen M, Dong Q, Wei Y, Chen Y, Ouyang K, Wei Z, Huang W (2019) Pathogenic characteristics of a porcine astrovirus strain isolated in China. Viruses. https://doi.org/10.3390/v11121156

37. Qin Y, Fang Q, Liu H, Ji C, Chen Y, Ouyang K, Wei Z, Huang W (2018) Construction of a reverse genetic system for porcine astrovirus. Arch Virol 163:1511-1518. https://doi.org/10.1007/ s00705-018-3771-4

38. Xiao CT, Luo Z, Lv SL, Opriessnig T, Li RC, Yu XL (2017) Identification and characterization of multiple porcine astrovirus genotypes in Hunan province, China. Arch Virol 162:943-952. https://doi.org/10.1007/s00705-016-3185-0

39. Lv SL, Zhang HH, Li JY, Hu WQ, Song YT, Opriessnig T, Xiao CT (2019) High genetic diversity and recombination events of porcine astrovirus strains identified from ill and asymptomatic pigs in 2017, Hunan Province, China. Virus Genes 55:673-681. https://doi.org/10.1007/s11262-019-01692-w

40. Cai Y, Yin W, Zhou Y, Li B, Ai L, Pan M, Guo W (2016) Molecular detection of Porcine astrovirus in Sichuan Province, China. Virol J 13:6. https://doi.org/10.1186/s12985-015-0462-6

41. Lan D, Ji W, Shan T, Cui L, Yang Z, Yuan C, Hua X (2011) Molecular characterization of a porcine astrovirus strain in China. Arch Virol 156:1869-1875. https://doi.org/10.1007/ s00705-011-1050-8

42. Mi S, Guo S, Xing C, Xiao C, He B, Wu B, Xia X, Tu C, Gong W (2020) Isolation and characterization of porcine astrovirus 5 from a classical swine fever virus-infected specimen. J Virol. https:// doi.org/10.1128/JVI.01513-20

43. Chen XU, He Y, Li W, Kalim U, Xiao Y, Yang J, Wang X, Yang S, Zhang W (2020) Identification and characterization of a novel recombinant porcine astrovirus from pigs in Anhui, China. Pol J Microbiol 69:471-478. https://doi.org/10.33073/pjm-2020-051

44. Zhao C, Chen C, Li Y, Dong S, Tan K, Tian Y, Zhang L, Huang J, Zhang L (2019) Genomic characterization of a novel recombinant porcine astrovirus isolated in northeastern China. Arch Virol 164:1469-1473. https://doi.org/10.1007/s00705-019-04162-8

45. Untergasser A, Cutcutache I, Koressaar T, Ye J, Faircloth BC, Remm M, Rozen SG (2012) Primer3-new capabilities and interfaces. Nucleic Acids Res 40:e115. https://doi.org/10.1093/nar/ gks596

46. De Benedictis P, Schultz-Cherry S, Burnham A, Cattoli G (2011) Astrovirus infections in humans and animals-molecular biology, genetic diversity, and interspecies transmissions. Infect Genet Evol 11:1529-1544. https://doi.org/10.1016/j.meegid.2011.07.024

47. Kumar S, Stecher G, Tamura K (2016) MEGA7: molecular evolutionary genetics analysis version 7.0 for bigger datasets. Mol Biol Evol 33:1870-1874. https://doi.org/10.1093/molbev/msw054

48. Shan T, Wang C, Tong W, Zheng H, Hua X, Yang S, Guo Y, Zhang W, Tong G (2012) Complete genome of a novel porcine astrovirus. J Virol 86:13820-13821. https://doi.org/10.1128/JVI. 02598-12

49. Su M, Qi S, Yang D, Guo D, Yin B, Sun D (2020) Coinfection and genetic characterization of porcine astrovirus in diarrheic piglets in China from 2015 to 2018. Front Vet Sci 7:462. https://doi.org/ $10.3389 /$ fvets.2020.00462

50. Zhang Q, Hu R, Tang X, Wu C, He Q, Zhao Z, Chen H, Wu B (2013) Occurrence and investigation of enteric viral infections in pigs with diarrhea in China. Arch Virol 158:1631-1636. https:// doi.org/10.1007/s00705-013-1659-x

51. Chen Q, Wang L, Zheng Y, Zhang J, Guo B, Yoon KJ, Gauger PC, Harmon KM, Main RG, Li G (2018) Metagenomic analysis of the RNA fraction of the fecal virome indicates high diversity in pigs infected by porcine endemic diarrhea virus in the United States. Virol J 15:95. https://doi.org/10.1186/s12985-018-1001-z

52. Li JS, Li MZ, Zheng LS, Liu N, Li DD, Duan ZJ (2015) Identification and genetic characterization of two porcine astroviruses from domestic piglets in China. Arch Virol 160:3079-3084. https://doi. org/10.1007/s00705-015-2569-x

53. Nagai M, Omatsu T, Aoki H, Otomaru K, Uto T, Koizumi M, Minami-Fukuda F, Takai H, Murakami T, Masuda T, Yamasato H, Shiokawa M, Tsuchiaka S, Naoi Y, Sano K, Okazaki S, Katayama Y, Oba M, Furuya T, Shirai J, Mizutani T (2015) Full genome analysis of bovine astrovirus from fecal samples of cattle in Japan: identification of possible interspecies transmission of bovine astrovirus. Arch Virol 160:2491-2501. https://doi.org/10. 1007/s00705-015-2543-7

Publisher's Note Springer Nature remains neutral with regard to jurisdictional claims in published maps and institutional affiliations. 